\title{
Experimental verification of the limits of optical channel intensity reciprocity
}

\author{
Dirk Giggenbach, ${ }^{1, *}$ William Cowley, ${ }^{2}$ Ken Grant, ${ }^{3}$ and Nicolas Perlot ${ }^{1}$ \\ ${ }^{1}$ Institute of Communications and Navigation, German Aerospace Center (DLR), \\ Oberpfaffenhofen, 82234 Wessling, Germany \\ ${ }^{2}$ Institute for Telecommunications Research, University of South Australia, Mawson Lakes SA 5095, Australia \\ ${ }^{3}$ Defence Science and Technology Organisation, Edinburgh SA 5111, Australia \\ ${ }^{*}$ Corresponding author: dirk.giggenbach@dlr.de
}

Received 10 February 2012; accepted 1 March 2012;

posted 6 March 2012 (Doc. ID 162904); published 22 May 2012

\begin{abstract}
Optical data links through the atmosphere suffer from turbulence-induced signal scintillation. In a coaxially-symmetric bidirectional link scenario, the variations of the axial intensities at both ends are correlated. This relation can be used as an inherent feedback mechanism, with negligible delay, to enhance the capacity of the transmission system. By experiment, we show the correlation coefficient of both received signals can reach values close to one over long atmospheric distances, provided the receiver apertures are smaller than specific intensity speckle structures, while the correlation reduces gradually with larger apertures. This allows transmission capacity to be optimized with adaptive transceiver systems that take into account the degree of correlation. (C) 2012 Optical Society of America
\end{abstract}

OCIS codes: $\quad 010.1300,060.2605$.

\section{Introduction}

Channel reciprocity in bidirectional fading channels connotes that the received signal levels at both link ends are similar or, ideally, identical. Perfectly correlated signals would mean that the transmitter has perfect and immediate knowledge of the fading at the other end of the link. This allows it to employ fade-mitigation techniques that reach towards theoretical channel capacity limits. These adaptive techniques include varying the data-rate, transmit power, modulation format, and/or code rate of forward error-correction systems. They have been widely studied in radio frequency transmission systems and, to a more limited extent, in free space optical (FSO) communication transmission [1,2]. In general, existing methods require a separate feedback channel to convey information regarding the current fade level at the receiver back to the adaptive

$1559-128 \mathrm{X} / 12 / 163145-08 \$ 15.00 / 0$

(C) 2012 Optical Society of America transmitter. This normally incurs a delay that impairs performance by an amount depending on the rate of channel variation compared to the feedback path delay.

In atmospheric optical links, the laser signals' wave-fronts are distorted by index-of-refraction turbulence (IRT) cells, which in the further propagation process, leads to self-interference. This produces intensity speckle patterns in the receiver plane, varying over space and time, with the movement of the atmosphere's IRT cells $[3,4]$. The size of these patterns is typically in the 10 to $100 \mathrm{~mm}$ range and is similar to a receiving aperture's diameter. The speckles typically change their shapes in milliseconds as the turbulence cells are moved by lateral wind, convection, and possibly, relative motion of mobile link partners. An optical receiver integrates the intensity over its receiving aperture, thus experiencing significant variations in received signal power over time. When the receiver's aperture size is significantly larger than the typical speckle pattern size, it integrates over several single speckles, which leads 
to smoothing of the received power vector both in variation strength and over time, an effect called aperture averaging [5-7].

When two transceivers are setup to send their signals towards each other through the same IRTvolume (i.e., transmitter- and receiver-apertures are coaxially aligned), the two beams will experience an identical IRT-structure, just in opposite directions. As the strength and size of the final scintillation patterns depend on the position of the specific IRT-cells in the propagation path, it will generally lead to different patterns at both ends with different strengths of scintillation (see Fig. 1).

However, it was theoretically predicted several years ago by Shapiro, Lutomirski, and Yura that in spherical wave propagation, the intensities should behave in a reciprocal manner for on-axis point transmitters and receivers [8,9]. Similar investigations-partly also for non-spherical waves-have been presented in [10] and [11]. The effect is also well known in LIDAR-applications, where the so-called backscatter-amplification beneficially contributes to increased surges of the reflected signal, which is detected through a receiver aperture close by or similar to the transmit aperture [12-14]. When reciprocity can be established reliably in an FSO communication link, instantaneous knowledge of signal variations at the other receiver is available, and accordingly compensation measures can be employed without a separate feedback channel, to increase the communication channel capacity.

Recently, measurement of reciprocity was reported in an aircraft to ground link $[15,16]$. There, correlation coefficients close to one could be derived from the signals measured between a $2 \times 2$ ground array of small tracked apertures and a remote terminal onboard an aircraft.

Related approaches not only aim to provide knowledge of signal variations at the other link end, but also try to correct IRT effects by adaptive optics techniques, to enable a stable and constant received signal through pre-distortion of the outgoing beam [17]. However, such systems are out of the scope of this paper.

While the on-axis intensity reciprocity has been theoretically and experimentally proven, the question remains to what extent the effect persists when enlarging the receive apertures to beyond what can

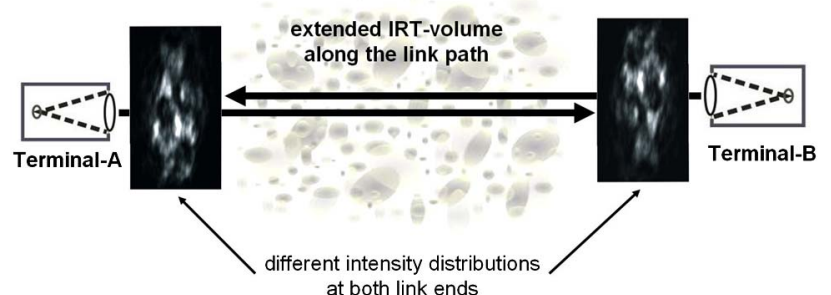

Fig. 1. (Color online) Bidirectional propagation through an extended volume of atmospheric turbulence cells generally results in different intensity distributions at both receiver planes. However, equal intensities exist exactly on-axis for spherical waves [요묘. be considered as close-to on-axis. When the link budget requires a certain receiver aperture to satisfy minimum average power, we expect reciprocity correlation to drop due to the integration over non-equal intensity distribution areas aside of the optical axis. At the same time, aperture averaging will take effect, beneficially reducing the variation in received power while the receiver diameter becomes larger. This causes power scintillation to drop to negligible values when employing very large aperture sizes.

In this paper we investigate experimentally the correlation of both received powers with aperture sizes in between those two extremes. This allows assessment of whether non-perfect reciprocity (i.e., reduced correlation coefficient) still allows beneficial application of adaptive transmission techniques.

For the relevant mathematical theory to our measurements, we reference [18], where an expression for the correlation coefficient in reciprocal transmission with finite sized apertures is derived and different application scenarios are evaluated numerically.

\section{Experimental Setup}

While the reciprocity is theoretically based on spherical waves, this is not a major limitation in most terrestrial FSO scenarios, as these links typically have relatively large Tx-divergence angles of several $100 \mu \mathrm{rad}$. This allows modelling as spherical waves in good approximation for the near-axis area. Beams can be considered as near-spherical waves when

$$
D_{\mathrm{Tx}} \ll \sqrt{\lambda \cdot L_{\mathrm{atm}}} .
$$

with transmit aperture diameter $D_{\mathrm{Tx}}$, wavelength $\lambda$, and atmospheric path length $L_{\text {atm }}[19]$. This condition is satisfied in our case (see Table 1 ), as we use Gaussian beams with FWHM-divergence angle of $550 \mu \mathrm{rad}$, and $L_{\text {atm }}$ equals the total link length (750 $\mathrm{m}$ to $20 \mathrm{~km})$.

Table 1. Parameters of the Experimental Setup Common to All Tests

\begin{tabular}{lcc}
\hline Parameter & Unit & Value \\
\hline $\begin{array}{l}\text { Center Wavelength/FWHM, Tx } \\
\text { of terminal "A" }\end{array}$ & $\mathrm{nm}$ & $1560 / 15$ \\
$\begin{array}{l}\text { Center Wavelength/FWHM, Tx } \\
\text { of terminal "B" }\end{array}$ & $\mathrm{nm}$ & $1555 / 12$ \\
$\begin{array}{l}\text { Tx-power in trials } A, B / C, D, E \\
\text { Tx-diameter, 1/e }\end{array}$ & $\mathrm{mW}$ & $10 \mathrm{~mW} / 26 \mathrm{~mW}$ \\
Tx-divergence, FWHM & $\mathrm{mm}$ & 2.1 \\
Outer Rx-aperture diameter $D_{o}$ & $\mu \mathrm{rad}$ & 550 \\
Inner Rx obscuration diameter & $\mathrm{mm}$ & 127 or 50 \\
$\quad D_{i}$ (caused by Tx-collimator) & & 26 \\
Effective Rx-area 127 mm & $\mathrm{m}^{2}$ & 0.0121 \\
Effective Rx-area 50 mm & $\mathrm{m}^{2}$ & 0.00143 \\
Focal length of Rx-Fresnel-lens & $\mathrm{mm}$ & $128 \mathrm{~mm}$ \\
Detector diameter (InGaAs-PIN) & $\mathrm{mm}$ & 1 \\
Amplification stages of detector & $\mathrm{V} / \mu \mathrm{W}$ & $20 \mathrm{~dB}: 0.0143 \mathrm{~V} / \mu \mathrm{W}$ \\
& & $30 \mathrm{~dB}: 0.0451 \mathrm{~V} / \mu \mathrm{W}$ \\
& & $40 \mathrm{~dB}: 0.143 \mathrm{~V} / \mu \mathrm{W}$ \\
& & $50 \mathrm{~dB}: 0.451 \mathrm{~V} / \mu \mathrm{W}$ \\
Sampling rate of Rx-power & Samples $/ \mathrm{s}$ & 1000 \\
\hline
\end{tabular}


One requirement for the experimental terminal setup was robustness, to be deployable ad hoc at several different locations in the field. We, therefore, chose a separately adjustable small Tx-collimator assembly with diameter $D_{i}$, positioned in the middle of the Rx-aperture (Fig. 2). This inner obscuration of the receiver aperture clearly prevented observation of axial speckle structure sizes below $D_{i}$, which has to be regarded later in the evaluation of reciprocity correlation.

The receive aperture diameter could be stopped down, from $127 \mathrm{~mm}$ to $50 \mathrm{~mm}$, which proved crucial to measure and quantify the channel reciprocity effect for extended apertures.

The two received signals were sampled locally at both link ends and their normalized correlation coefficient $(\rho$, also denoted CCF for correlation coefficient in Fig. 5) and further link parameters were calculated using

$$
\rho=\frac{E\left\{\left(h_{1}-\mu_{1}\right)\left(h_{2}-\mu_{2}\right)\right\}}{\sigma_{1} \sigma_{2}},
$$

where $h_{1}$ and $h_{2}$ represent the two received signal amplitudes at either end of the link, and $\sigma_{i}$ and $\mu_{i}$ are their standard deviations and means respectively. In practice, two time series were recorded asynchronously and then post-processed to synchronize them with respect to a reference which had been imposed on the signals.

\section{Link Paths and Path Profiles}

All measurements reported here were executed at various locations in and near Adelaide, South Australia, between February and April 2011, as shown in Fig. 3. Five different transmission scenarios were used, from $750 \mathrm{~m}$ up to $20 \mathrm{~km}$ distance, from horizontal near-ground to elevated links between mountaintop and a building-roof at lowland.

Paths $A$ and $B$ were horizontal and near ground at the optical range facility of Defence Science and Technology Organisation) (DSTO). Turbulence strength, represented by index of refraction struc-

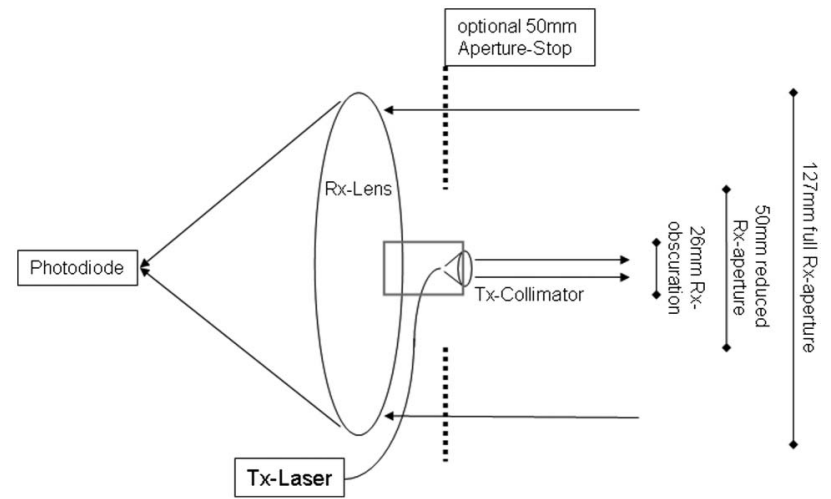

Fig. 2. Geometry of both transceiver terminals which were installed with tip-tilt-tables on geodetic tripods. Position of photodiode and Tx-direction could be adjusted separately to allow co-alignment of Tx-beam with Rx-field-of-view. As Tx-lasers MQW-type laser diodes were used (PL15 from PD-LD Inc.).

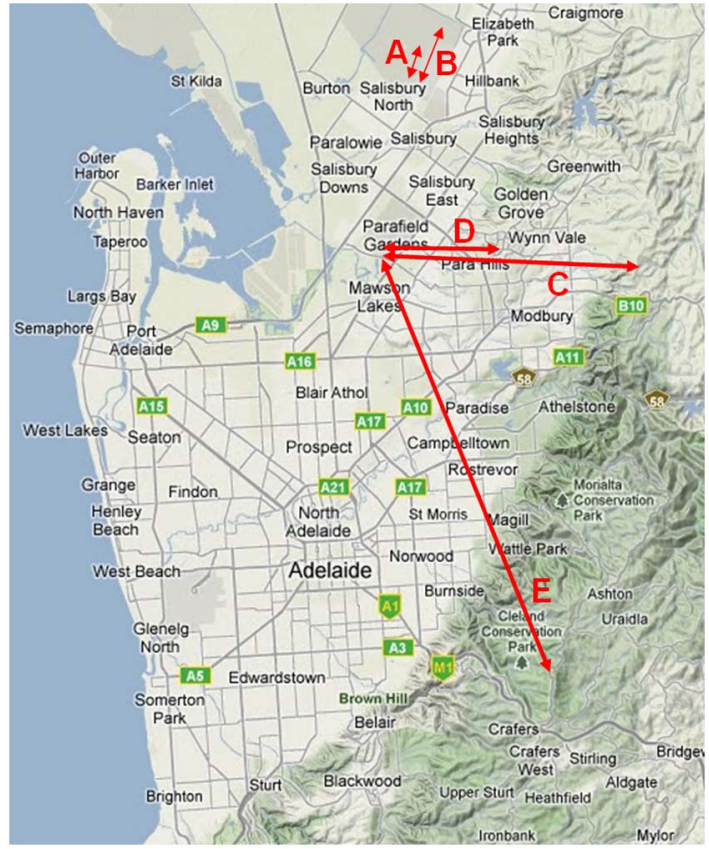

Fig. 3. (Color online) Map of measurement paths at Adelaide, South Australia (underlying Map data @2011 Google, Whereis®, Sensis Pty Ltd.).

ture parameter $C_{n}^{2}$, was measured with a commercial scintillometer (Scintec BL900) (see Table 2). Paths $C$ through $E$, however, led from a rooftop to various hills, generally causing increased height above ground in the middle of the link. As the IRT-strength (represented by $C_{n}^{2}$ ) decreases with height according to various models, one can estimate a $C_{n}^{2}$-profile from the height above ground.

It is intuitive that the special symmetric case of a dominating IRT-passage in the middle of the link would lead to similar intensity patterns at both ends. However, in order to investigate the more general situation, which is of greater practical interest, we had to make sure that the continuous IRT profile did not bear such a dominating middle portion. This can be verified by calculating the weighted $C_{n}^{2}$-profile from the height above ground [20].

First, we need to calculate the $C_{n}^{2}$-profile from the height above ground. As here we had near-ground (few meters to $300 \mathrm{~m}$ above ground) and nearly horizontal paths, the $C_{n}^{2}$-profile models popular in astronomy-like Hufnagel-Valley — could not be used as they insufficiently model the atmospheric turbulence close to ground. Instead, we need here a model that covers the region in the atmospheric boundary layer below the first inversion layer. From [4], and [20], the IRT in this region should behave according to

$$
C_{n}^{2}(h)=C_{n}^{2}\left(h_{0}\right) \cdot\left(\frac{h}{h_{0}}\right)^{-k},
$$

with $h$ smaller than the height of the atmospheric inversion layer and $h_{0}$ larger than some meters. For sunny daytime with an unstable boundary layer, $k$ should be $4 / 3$, but should drop to around $2 / 3$ during the more stable situations around sunrise, short 


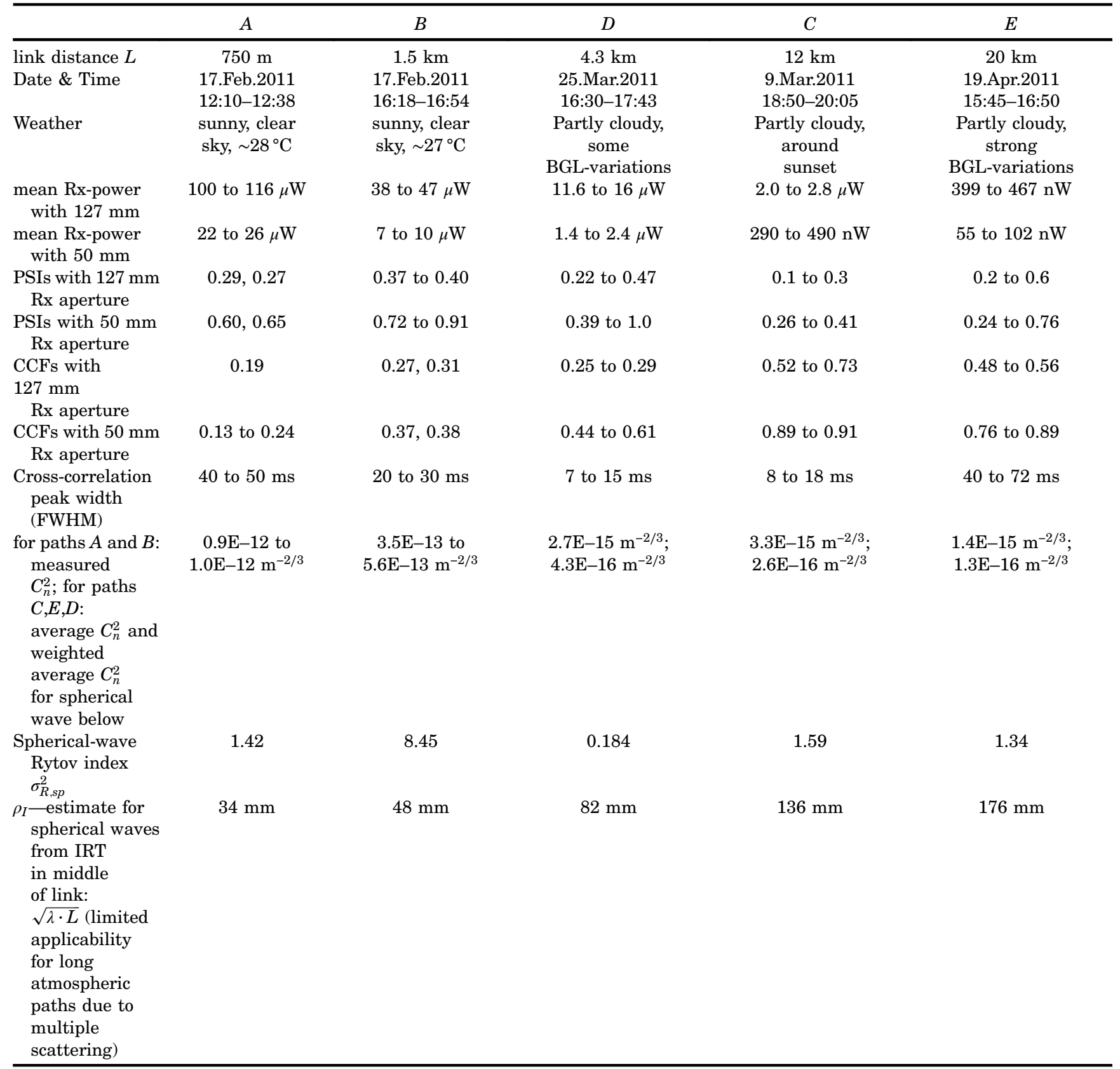

before sunset, and during night. As our experiments $C$ through $E$ took place either near sunset or on a partly cloudy afternoon, we assume here $k=1$. Typical values of $C_{n}^{2}(9 \mathrm{~m}) \sim 10^{-14} \mathrm{~m}^{-2 / 3}$ can be deduced from Fig. 2.7 in [20]. Thus, as $C_{n}^{2}(h)$-profile here we used $C_{n}^{2}(h)=\overline{9} * 10^{-14} \mathrm{~m}^{1 / 3} / \mathrm{h}$.

Because we used a fairly strong divergence angle of $550 \mu \mathrm{rad}$ FWHM, the wave can be approximated as a spherical wave. The scintillation strength can be estimated from the Rytov variance $\sigma_{R, \mathrm{sp}}^{2}$ for a spherical wave [20]:

$$
\sigma_{R, \mathrm{sp}}^{2}=2.25 k^{7 / 6} L^{5 / 6} \int_{0}^{L} C_{n}^{2}(z) w(z) \mathrm{d} z,
$$

where $w(z)$ is given by

$$
w(z)=\left(\frac{z}{L}\right)^{5 / 6}\left(1-\frac{z}{L}\right)^{5 / 6}
$$

The function $w(z)$ can be interpreted as a weighting function for $C_{n}^{2}$ over the path distance $z$ and is also plotted in the profiles of paths as shown in Fig. $\underline{4}$.

\section{Measurement Results}

Bidirectional link tests as depicted in Fig. 1 were performed over all five distances. Figure 5 shows typical received power vectors at both ends for four different situations.

A summary of several measured link parameters is given in Table 2. Correlation coefficients observed with the small aperture $(50 \mathrm{~mm})$ were between 0.76 
and 0.91 for the long ranges while with the large aperture $(127 \mathrm{~mm})$ reached between 0.48 and 0.73 . In Fig. 6 all reliable measurements of $\rho$ are plotted versus the square root of the link distance. This shows the increase of $\rho$ with range extrapolated up to $\sim 15 \mathrm{~km}$. The development is more explicit for the smaller aperture, while the ratio between aperture and speckle size varies more strongly with the larger aperture. According to the trend line for $50 \mathrm{~mm}$ aperture drawn in Fig. $6, \rho$ is expected to saturate towards unity around $1 \overline{5} \mathrm{~km}$ with this smaller aperture. We could not perform measurements with exactly $15 \mathrm{~km}$ distance, and our measurements at the $20 \mathrm{~km}$ path showed reduced signal-to-noise ratio (SNR) due to the high range loss. In addition, it is believed that the scintillation speckle pattern sizes are likely to reduce at such long horizontal paths due to multiple scattering, making even the $50 \mathrm{~mm}$ aperture too large to observe the correlation properly at very long distances. However, this behavior will depend heavily on the IRT-profile, making the $15 \mathrm{~km}$ not a specific limiting distance of the channel reciprocity with $50 \mathrm{~mm}$.

\section{Interpretation of Findings}

According to general scintillation theory for propagation through IRT, the final strength of the intensity scintillation depends on the position of (or distance from) the scattering IRT-volume. In the case of spherical waves, however, the symmetry in the weighting
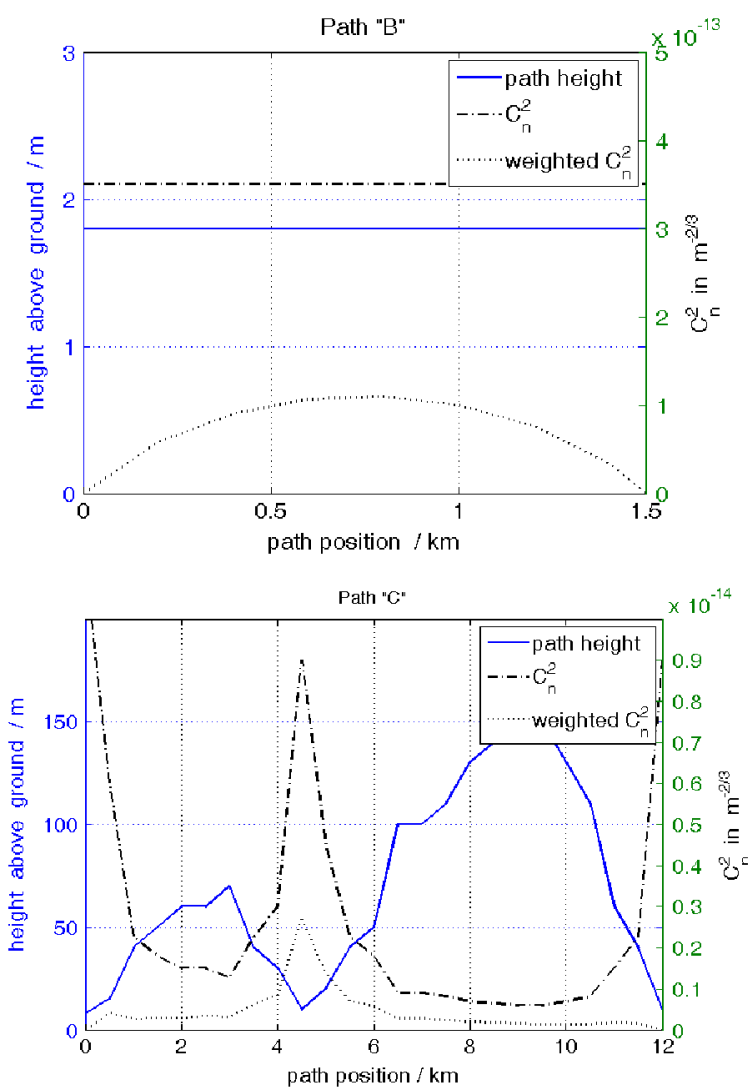

Fig. 4. (Color online) Height-above-ground profiles (solid lines), $C_{n}^{2}$-profile (dot-dash lines), and weighted $C_{n}^{2}$-profilew $(z) * C_{n}^{2}(z)($ dotted lines, weighted with $w(z)$ of a spherical wave) of propagation paths $B, D, C, E$ ( $A$ is similar to $B$, only half of its path length). function (5) of the turbulence over path, results in the same influence of on-axis scintillation strength from IRT that has a certain distance from the Tx or from the $\mathrm{Rx}$.

Furthermore, the specific intensity pattern size produced by a continuous turbulent volume depends on the positions where the individual turbulent scattering is located. This means that scattering events far from the Rx will produce large speckle sizes, while those close to the Rx generate small speckles sizes. Only when there is enough propagation distance for the path close to the Rx to still produce a large enough speckle (at least as large as the Rx-diameter), it can contribute beneficially to a high correlation coefficient of both signals. But, as the weighting function (므) suppresses IRT close to Rx and Tx, the too-small speckles from volumes close to the $R x$ have minor effect on the scintillation than those from closer to the middle of the link. Using the Fresnel-size $\sqrt{\lambda \cdot L}$ as an approximation of speckle size in weak-turbulence scenarios with spherical waves [19], one can estimate the speckle structure sizes originating from various positions along the link. Weighted with (5), structure sizes around $\sqrt{\lambda \cdot L}$, which are caused by IRT near the middle of the link (see Table 1 in [18]), should dominate the intensity patterns at both ends, while those from IRT closer to or further from the respective $\mathrm{Rx}$ are under-developed. Thus, we expect only apertures that are smaller than $\sqrt{\lambda \cdot L}$ to show a significant correlation effect. The according values are given in the last
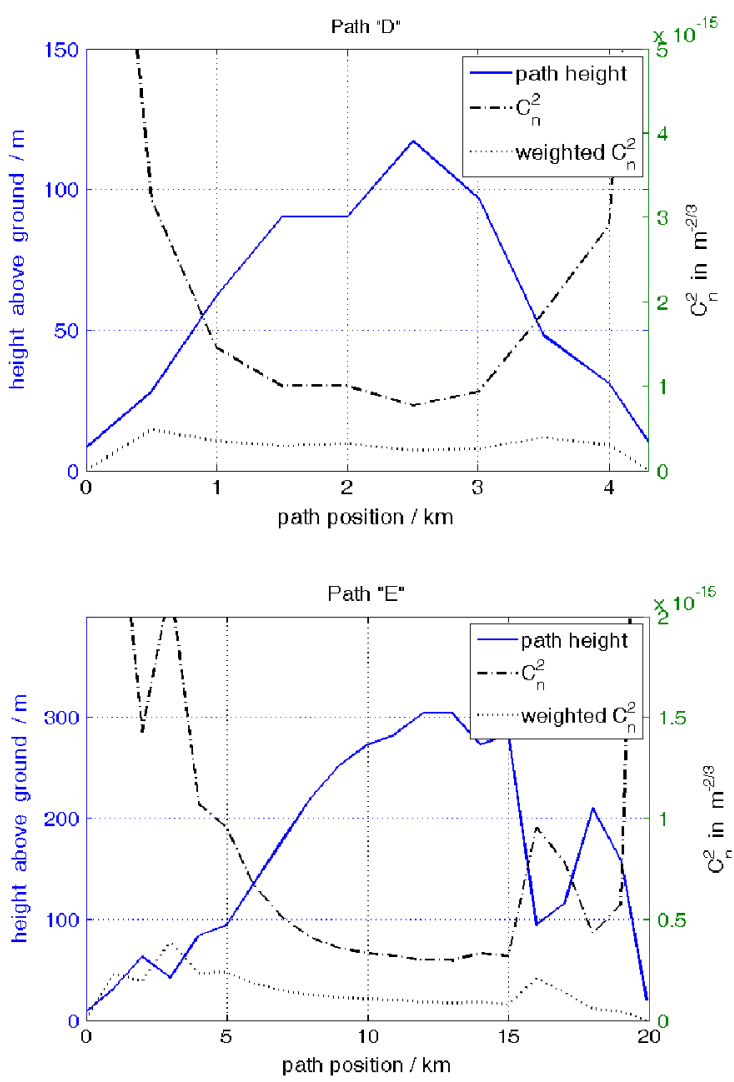

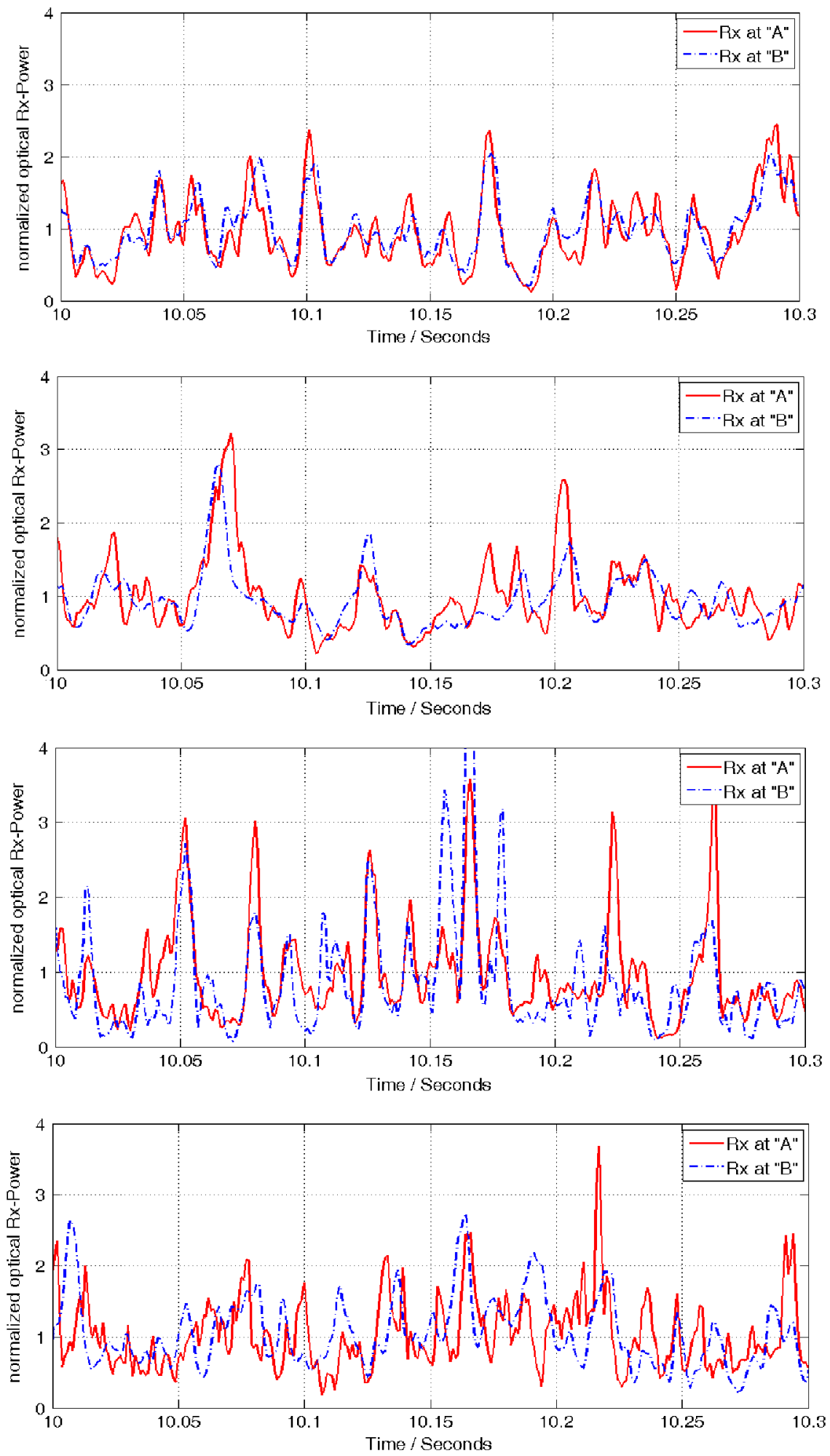

Fig. 5. (Color online) Typical simultaneously measured normalized received power vectors. From top down: Path "C" with 50 mm aperture and $\mathrm{CCF}=0.91$, Path " $C$ " with $127 \mathrm{~mm}$ aperture and $\mathrm{CCF}=0.74$, Path " $D$ " with $50 \mathrm{~mm}$ aperture and $C C F=0.61$, and path " $D$ " with $127 \mathrm{~mm}$ aperture and $\mathrm{CCF}=0.27$. Rx-A solid line, Rx-B dashed line, $300 \mathrm{~ms}$ of Rx data samples each.

row of Table $\underline{2}$ as speckle size estimate $\rho_{I}$. It can be assumed that the Fresnel-size approximation overestimates the real speckle size at long distances, because multiple scattering along the propagation path prevents intensity structure sizes from further increasing with propagation distance. Thus, even smaller apertures than those stated in Table $\underline{2}$ will be required to achieve high correlation.

Further to this simple estimation for the reciprocity-area, in [18] an analytical derivation for 


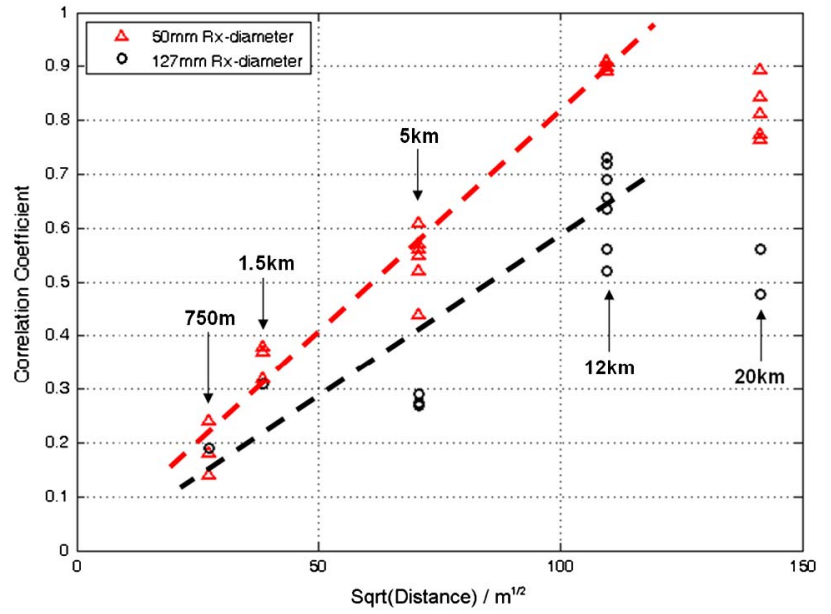

Fig. 6. (Color online) Correlation coefficients versus square root of link distance. There is an approximately linear increase in CCF with the square root of the range, up to $\sim 15 \mathrm{~km}$. The CCF-saturation to 1 should be expected around $15 \mathrm{~km}$ when the aperture becomes significantly smaller than the speckle size. It is believed that this situation could not be reached even with the long path $E(20 \mathrm{~km})$ because multiple scattering prevented reciprocal speckle structures to increase further with distance.

reciprocity-strength is given which we discuss in the following:

Fig. 7 uses the same CCF-data as Fig. 6 , but sorts it over the ratio of the receive aperture diameter to $\sqrt{\lambda \cdot L}$. Here, we observe that this ratio can be increased to close to unity until significant reduction of correlation takes place. This finding expands the predication from on-axis reciprocity to receiver diameters below Fresnel-size.

The continuous progression of CCF is plotted in Fig. 3 of [18]. With an unobstructed receiver aperture and for ratios of $D_{\mathrm{Rx}} / \sqrt{\lambda \cdot L}$ smaller than unity, a CCF of higher than 0.65 can be expected. For our experimental case with an inner obscuration, values are slightly lower. The latter is obvious considering that most correlation is generated by the on-axis fraction of intensity. By omitting this part through inner obscuration with $D_{i}$, a small but important part of the speckle pattern can not positively contribute to the CCF. When speckle size is small, i.e., close to $D_{i}$, this missing intensity area will cause cognizable reduction of the CCF. In Fig. 7 the theoretical values for both cases (with and without inner obscuration) calculated based on the $C_{n}^{2}$-profiles from Fig. 4, are shown in comparison with the measured data. This plot together with the findings of [18] supports the conclusion that channel reciprocity with finite sized receiver apertures scales with the ratio of aperture diameter over the Fresnel-size approximation for speckle size, $\sqrt{\lambda \cdot L}$.

\section{Summary, Limitations, and Potential Applications}

These measurements quantify the effect of channel intensity reciprocity in atmospheric bidirectional laser links with finite aperture sizes. Practical limitations of the measurement setup (inner-obscuration of the Rx-aperture, and minimum aperture size lim- ited to $50 \mathrm{~mm}$ ) have prevented to reach correlation coefficients closer to unity than 0.91 . However, in [15] it has been demonstrated that values of 0.99 can be reached when even smaller aperture diameters (there $25 \mathrm{~mm}$ ) are employed. From our measurements, it can be expected that the reciprocity of intensity is limited to an axial intensity area comparable to the Fresnel-size (which serves as approximation of speckle-size), while the intensity patterns further from the link axis deviate in structure from each other. Thus, only link geometries with apertures smaller than the single speckle size $\rho_{I}$ should be expected to achieve high correlation coefficients. In our tests, such aperture sizes were limited to few centimeters.

The general applicability in much longer links, or strongly asymmetric links, or links with high lateral wind speed needs still to be evaluated. It would be valuable to verify if the effect can be beneficially used in extremely asymmetric IRT-profiles, such as satellite to ground links. Especially for future optical geostationary feeder links exploitation of this effect could prove very beneficial: instead of waiting for a negative acknowledge from the GEO-satellite when data has been lost during a fade (and causing the typical delays of several $100 \mathrm{~ms}$ ), the transmitter on ground could directly resend data when a fade was observed. In this application, the uplink can be adaptive, but not the downlink because propagation delay between the atmosphere and the satellite is longer than the IRT correlation time. Additionally, as in a satellite-link scenario the point-ahead angle between receive- and transmit-signal is often larger than the

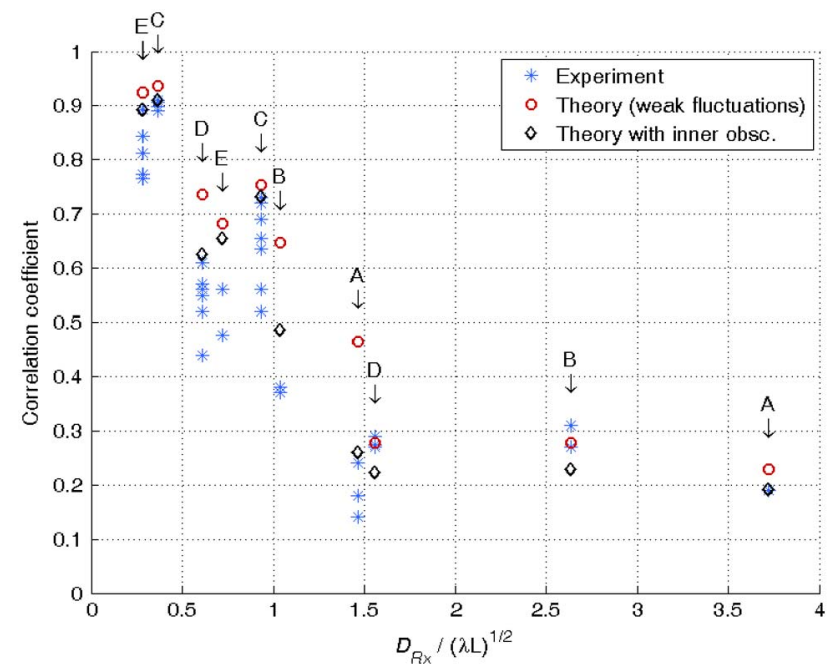

Fig. 7. (Color online) Correlation coefficient versus receiver aperture diameter normalized by the Fresnel size $\sqrt{\lambda \cdot L}$. The correlation values increase significantly when the ratio is below one. Measured values (asterisks) are compared with theoretical values without an inner obscuration (circles) and regarding the inner obscuration of $26 \mathrm{~mm}$ in our experiment (diamonds). The theoretical values are calculated according to [18]. The scenario letters ( $A$ to $E$ ) are indicated on top of each set of values, where each scenario provides two sets due to the two aperture sizes. 
isoplanatic angle of the atmosphere, limitations in applicability must be expected.

Another auspicious future link scenario is longrange inter-platform links between High Altitude Platforms (HAP), which can be used as communications nodes in the stratosphere. Due to the nature of these link geometries (long distances with continuous IRT and large speckle sizes at the receiver), the reciprocity should be well developed, allowing efficient application of mitigation techniques.

The most promising application, however, should be long-range bidirectional inner-atmospheric FSO links using common Tx- and Rx-apertures and having a requirement for using small aperture sizes (smaller than $\sim 50 \mathrm{~mm}$ for $1550 \mathrm{~nm}$ wavelength), e.g., due to size or cost constraints. In such links, adaptive forward error correction, or variable datarate schemes could be used to maximize throughput and minimize losses, while allowing longer distances through optimum fading compensation. Admittedly, whenever larger Rx-apertures can be used, the fading-reduction from aperture-averaging (besides the even higher mean power) will supersede the benefit from channel reciprocity.

We are not aware yet of an application-verification of the reciprocity effect for communication link quality improvement, by varying transmit power, or datarate, or coding, according to the measured Rx-power. Investigations of beneficial applications based on adaptive data-rate are ongoing.

We gratefully acknowledge the support of Hidayat Soetiyono and Terry Kemp of the Institute for Telecommunications Research at University of South Australia. The research stay of Dirk Giggenbach at the University of South Australia was financed through the Research Semester Grant of the German Aerospace Center (DLR).

\section{Appendix A: Abbreviations and Symbols:}

\begin{tabular}{cl}
\hline ACOV & Auto Covariance function \\
BGL & Background Light \\
$C_{n}^{2}$ & structure constant of optical turbulence \\
FSO & Free Space Optical communications \\
FWHM & Full-Width Half-Maximum \\
IRT & Index-of-Refraction Turbulence \\
PSI & Power Scintillation Index \\
Rx & receiver \\
Tx & transmitter \\
XCOV & Cross Covariance function \\
$\rho$ & normalized correlation coefficient, also denoted CCF \\
$\rho_{I}$ & size of intensity structure \\
$\sigma_{R}$ & Rytov variance \\
\hline
\end{tabular}

\section{References}

1. I. B. Djordjevic, "Adaptive modulation and coding for freespace optical channels," J. Opt. Commun. Netw. 2, 221-229 (2010).

2. S. Bloom, E. Korevaar, J. Schuster, and H. Willebrand, "Understanding the performance of free-space optics [Invited]," J. Opt. Commun. Netw. 2, 178-200 (2003).

3. L. C. Andrews and R. L. Phillips, Laser Beam Propagation through Random Media 2nd ed. (SPIE Press, 2005).

4. J. W. Strohbehn, ed., Laser Beam Propagation in the Atmosphere (Springer,1978).

5. L. C. Andrews, "Aperture-averaging factor for optical scintillations of plane and spherical waves in the atmosphere," J. Opt. Soc. Am. A 9, 597-600 (1992).

6. J. H. Churnside, "Aperture averaging of optical scintillation in the turbulent atmosphere," Appl. Opt. 30, 1982-1994 (1991).

7. M. A. Khalighi, N. Aitamer, N. Schwartz, and S. Bourennane, "Turbulence mitigation by aperture averaging in wireless optical systems," in ConTEL 2009, 10th International Conference on Telecommunications (IEEE, 2009), pp. $59-66$.

8. R. F. Lutomirski and H. T. Yura, "Propagation of a finite optical beam in an inhomogeneous medium," Appl. Opt. 10, 1652-1658 (1971).

9. J. H. Shapiro, "Reciprocity of the turbulent atmosphere," J. Opt. Soc. Am. 61, 492-495 (1971).

10. J. H. Shapiro, "Optimal power transfer through atmospheric turbulence using state knowledge," IEEE Trans. Commun. Technol. 19, 410-414 (1971).

11. D. L. Fried and H. T. Yura, "Telescope-performance reciprocity for propagation in a turbulent medium," J. Opt. Soc. Am. 62, 600-602 (1972).

12. V. A. Banakh and V. L. Mironov, Lidar in a Turbulent Atmosphere (Artech House, 1987).

13. J. F. Holmes, "Enhancement of backscattered intensity for a bistatic lidar operating in atmospheric turbulence," Appl. Opt. 30, 2643-2646 (1991).

14. V. A. Banakh, I. N. Smalikho, and C. Werner, "Numerical simulation of the effect of refractive turbulence on coherent lidar return statistics in the atmosphere" Appl. Opt. 39, 5403-5414 (2000).

15. R. R. Parenti, J. M. Roth, J. Shapiro, and F. G. Walther, "Observations of channel reciprocity in optical free-space communications experiments," in Applications of Lasers for Sensing and Free Space Communications, OSA Technical Digest (CD) (Optical Society of America, 2011).

16. F. G. Walther, S. Michael, R. R. Parenti, and J. A. Taylor "Air-to-ground lasercom system demonstration design overview and results summary," Proc. SPIE 7814, 78140Y (2010).

17. J. D. Barchers and D. L. Fried, "Optimal control of laser beams for propagation through a turbulent medium," J. Opt. Soc. Am. A 19, 1779-1793 (2002).

18. N. Perlot and D. Giggenbach, "Scintillation correlation between forward and return spherical waves," Appl. Opt. (to be published)

19. L. C. Andrews and R. L. Phillips, Laser Beam Propagation Through Random Media (SPIE Press, 1998), p. 186.

20. F. G. Smith, ed., The Infrared \& Electro-Optical Systems Handbook, Volume 2: Atmospheric Propagation of Radiation, 2nd ed. (SPIE-Press, 1996). 